5 Diel R, Loddenkemper R, Zellweger JP, et al. Old ideas to innovate tuberculosis control: preventive treatment to achieve elimination. Eur Respir J 2013; 42: 785-801.

6 Voniatis C, Migliori GB, Voniatis M, et al. Tuberculosis elimination: dream or reality? The case of Cyprus. Eur Respir J 2014; 44: 543-546.

7 D'Ambrosio L, Dara M, Tadolini M, et al. Tuberculosis elimination: theory and practice in Europe. Eur Respir J 2014; 43: 1410-1420.

8 Zvada SP, Denti P, Donald PR, et al. Population pharmacokinetics of rifampicin, pyrazinamide and isoniazid in children with tuberculosis: in silico evaluation of currently recommended doses. J Antimicrob Chemother 2014; 69: 1339-1349.

9 Codecasa LR, Ciconali G, Mazzola E, et al. Managing an extensively drug-resistant tuberculosis outbreak: the public health face of the medal. Eur Respir J 2015; 45: 292-294.

\title{
CPAP holiday: are we there yet?
}

To the Editor:

We appreciated the article by Rossi et al. [1] entitled "Is continuous positive airway pressure necessarily an everyday therapy in patients with obstructive sleep apnoea?", as well as the corresponding editorial [2], recently published in the European Respiratory Journal. Indeed, there are limited data on the evolution of obstructive sleep apnoea (OSA) during continuous positive airway pressure (CPAP) therapy and whether this treatment is required on a daily basis.

Although as Rossi et al. [1] freely admitted, their study was not without flaws, we feel that some issues should be further addressed and discussed. The use of pulse oximetry to diagnose sleep apnoea is not recommended [3], as it may underestimate the degree of the disease, which was exactly what was being studied in this case. In addition, it is possible that the increase in the Epworth Sleepiness Scale score observed in group 2 patients independently of OSA recurrence might be explained by respiratory events and sleep arousals not diagnosed by that examination. Thus, this stands as an important limitation.

As from the 125 patients enrolled in the study, only nine (7\%) patients did not have OSA recurrence after 2 weeks (in 89 patients, it recurred within 4 days and those patients were not further evaluated), we think that the overall conclusions are excessively optimistic. Additionally, we would like to inquire whether the patients who stopped CPAP treatment and did not have a return of OSA even after 2 weeks $(n=9)$ had a greater reduction in weight since the beginning of treatment than other patients. No reference to this possible variation is made in the article and we feel this is highly relevant. Concerning the CPAP treatment, we wonder if the pressure used in those patients was significantly lower than in those in whom OSA returned.

As there are no clear recommendations in clinical practice regarding for how long CPAP should be suspended before evaluating OSA persistence in order to stop the therapy, as for example, after a significant loss of weight, we feel that this study could be of guidance. It allows us to admit that it could be prudent only to perform the sleep evaluation 2 weeks after withdrawing CPAP, as $55 \%$ of patients that did not have OSA recurrence after 4 days had more than 10 events per hour at the 2 -week evaluation, and this should be highlighted.

The hypothesis of the study was based on reports of various changes in the upper airway (inflammation, oedema, trauma to tissues, etc.) in patients with OSA, and the assumption that CPAP reduced the palatal "clatter and bang" of untreated OSA [2]. Although the physiological processes behind it are perceptible, there is no evidence this it actually occurred in those cases; for the time being, it is only speculation.

The authors suggested that a more flexible approach to CPAP use might improve overall compliance. This is a very delicate and important point. Recent studies have showed very good adherence and compliance to CPAP treatment [4-6], while others have reported that those who have good compliance in the initial days are more likely to have long-term adherence $[4,7]$.

Few head-to-head trials have been performed evaluating differences in adherence to daily drugs and intermittent dosing schedules, as most comparative trials of intermittent and daily agents focused on efficacy rather than adherence [8]. Adherence seemed better with intermittently dosed agents than daily dosed agents under the same conditions $[8,9]$. Nevertheless, it is widely recognised that data for oral drug 
compliance may not be generalised to all kinds of therapy, as it seems that adherence to therapy may vary across different pathologies and therapeutic approaches [10].

Thus, regarding intermittent CPAP use, not only is information to believe it could improve adherence scarce, but also, it could even diminish patients' perception of the importance of regular use, deteriorating previous strategies developed to enhance adherence [6], so caution should be advised until further studies have been conducted.

@ERSpublications

Information to believe intermittent CPAP could improve adherence is scarce http://ow.ly/DgZAi

Mafalda van Zeller $^{1}$ and Marta Drummond ${ }^{1,2}$

${ }^{1}$ Dept of Pulmonology, Centro Hospitalar de São João, Porto, Portugal. ${ }^{2}$ Faculty of Medicine, University of Porto, Porto, Portugal.

Correspondence: Mafalda van Zeller, Dept of Pulmonology, Centro Hospitalar de São João, Alameda Professor Hernâni Monteiro, 4200-319 Porto, Portugal. E-mail: vanzeller.mafalda@gmail.com

Received: July 202014 | Accepted: July 302014

Conflict of interest: None declared.

\section{References}

1 Rossi VA, Schwarz EI, Bloch KE, et al. Is continuous positive airway pressure necessarily an everyday therapy in patients with obstructive sleep apnoea? Eur Respir J 2014; 43: 1387-1393.

2 Strohl KP, Wellman A. When CPAP is stopped: what are the "on switches" of sleep apnoea? Eur Respir J 2014; 43: 1227-1229.

3 Epstein LJ, Kristo D, Strollo PJ Jr, et al. Clinical guideline for the evaluation, management and long-term care of obstructive sleep apnea in adults. J Clin Sleep Med 2009; 5: 263-276.

4 van Zeller M, Severo M, Santos AC, et al. 5-years APAP adherence in OSA patients - do first impressions matter? Respir Med 2013; 107: 2046-2052.

5 McArdle N, Devereux G, Heidarnejad H, et al. Long-term use of CPAP therapy for sleep apnea/hypopnea syndrome. Am J Respir Crit Care Med 1999; 159: 1108-1114.

6 Soares Pires F, Drummond M, Marinho A, et al. Effectiveness of a group education session on adherence with APAP in obstructive sleep apnea - a randomized controlled study. Sleep Breath 2013; 17: 993-1001.

7 Budhiraja R, Parthasarathy S, Drake CL, et al. Early CPAP use identifies subsequent adherence to CPAP therapy. Sleep 2007; 30: 320-324.

8 Kruk ME, Schwalbe N. The relation between intermittent dosing and adherence: preliminary insights. Clin Ther 2006; 28: 1989-1995.

9 Cooper C. Beyond daily dosing: clinical experience. Bone 2006; 38: Suppl. 1, S13-S17.

10 Yeaw J, Benner JS, Walt JG, et al. Comparing adherence and persistence across 6 chronic medication classes. J Manag Care Pharm 2009;15:728-740.

From the authors:

We appreciate the interest shown by M. van Zeller and M. Drummond in our paper [1], who suggest a more cautious interpretation regarding the possibility of withdrawing continuous positive airway pressure (CPAP) therapy for short periods and raise some concerns about how to select candidates properly who might safely undergo this.

In our study, we observed that about one-third of patients affected by obstructive sleep apnoea (OSA) syndrome and treated with CPAP did not experience recurrence of oxygen desaturations after four nights of CPAP therapy withdrawal, and about $10 \%$ did not after 2 weeks [1]. Therefore, we put forward the hypothesis that some patients may be able to stop CPAP for short periods without recurrence of OSA and its related pathophysiological consequences. As already addressed in our article [1], we agree that the use of pulse oximetry and its derivatives (oxygen desaturation index (ODI)) to assess sleep-disordered breathing has its limitations, although most researchers have found near equivalence of apnoea-hypopnoea index (AHI) to ODI. Neither conventional AHI measurements nor oximetric derivatives capture sleep 